\title{
Schweiz ohne Beamte - Staatsdiener ohne Pension? Zur Alterssicherung des eidgenössischen Bundespersonals
}

Friso Ross

I. Grundlegung

1. Bundespersonal als Vergleichsgruppe

2. Rechtliche Stellung des Bundespersonals

3. Alterssicherung des Bundespersonals im Kontext allgemeiner sozialer Sicherung

II. Ausgestaltung der Alterssicherung des Bundespersonals

1. Das Drei-Säulen-Konzept der Alterssicherung

2. Die AHV als Erste Säule und Basissicherung

a) Leistungen der AHV: Minimal- und Maximalrente

b) Finanzierung der AHV

3. Berufliche Vorsorge als Zweite Säule

a) Einrichtungen der Beruflichen Vorsorge des Bundespersonals

b) Leistungen der Beruflichen Vorsorge: Kapitalgedeckte Altersrente

c) Leistungen der Beruflichen Vorsorge: Kapitalabfindung

d) Finanzierung der Beruflichen Vorsorge für das Bundespersonal

e) Sicherung der Beruflichen Vorsorge für das Bundespersonal

III. Bewertung 


\section{Grundlegung}

\section{Bundespersonal als Vergleichsgruppe}

Die dem Bundesrat als Haupt der Exekutive unterstehende Bundesverwaltung wird durch die Bundeskanzlei, durch die einzelnen Departements und ihre Ämter sowie durch dezentralisierte Verwaltungseinheiten wahrgenommen. ${ }^{1} \mathrm{Zu}$ letzteren gehören etwa Swissmedic, das Schweizerische Heilmittelinstitut, oder das Eidgenössische Institut für Geistiges Eigentum.

In der Bundesverwaltung ist das Bundespersonal tätig. Unter den Begriff des Bundespersonals fallen alle Personen, die in einem Arbeitsverhältnis zum Bund stehen und somit einer personalrechtlichen Regelung des Bundes unterliegen. ${ }^{2}$ Zum Bundespersonal in einem solchen Sinne gehören nicht die Magistratspersonen; dies sind die Mitglieder des Bundesrates, die ordentlichen Richter des Bundesgerichts und der Bundeskanzler. ${ }^{3}$

\section{Rechtliche Stellung des Bundespersonals}

Innerhalb des Bundespersonals wurde vor den Reformen der 1990er Jahre zwischen Beamten und Angestellten unterschieden. Herausragendes und praktisch einziges Unterscheidungsmerkmal war die Wahl des Beamten für eine mehrjährige Amtsdauer, ${ }^{4}$ nach deren Ablauf das Dienstverhältnis endete. Faktisch wie rechtlich kehrte sich dieses Amtsdauerprinzip im Laufe der Zeit in sein Gegenteil um, indem das Dienstverhältnis des Beamten nach Ablauf der Amtsdauer nur aus sachlichem Grund beendet werden durfte und damit ein bedingter Anspruch auf Wiederwahl entstand. ${ }^{5}$

1 Art. 2 Abs. 1-3 Regierungs- und Verwaltungsorganisationsgesetz (RVOG) vom 21. März 1997 (SR 172.010). Im Folgenden werden alle Normen des Bundes, wie in der Schweiz üblich, nach der offiziellen Systematischen Sammlung des Bundesrechts (SR) zitiert. Gesetzesrevisionen werden nach dem Bundesblatt der Schweizerischen Eidgenossenschaft (BBl) zitiert. Beide Quellen sind online verfügbar: Die SR unter http://www.admin.ch/ch/d/sr/sr.html und das BBl unter http://www.admin.ch/ch/d/ff/index.html.

2 Jaag/Müller/Tschannen, Ausgewählte Gebiete des Bundesverwaltungsrechts, 6. Aufl. 2006, S. 3.

3 Legaldefinition nach Art. 1 Abs. 1 Bundesgesetz über Besoldung und berufliche Vorsorge der Magistratspersonen vom 6. Oktober 1989 (SR 172.121).

4 Helbling, Totalrevision des eidgenössischen Beamtengesetzes - eine rechtliche Auslegeordnung, ARP 1993, S. 647, 660.

5 Michel, Amtsdauersystem, in: Helbling/Poledna (Hrsg.), Personalrecht des öffentlichen Dienstes, 1999, S. $155,165$. 
Das heutige Recht des Bundespersonals ${ }^{6}$ kennt vorgenannte Unterscheidung in Beamte und Angestellte nicht mehr. ${ }^{7}$ Das Bundespersonal besteht aus Angestellten. ${ }^{8}$ Es gibt grundsätzlich keine Bundesbeamten mehr. ${ }^{9}$ Auch auf Zeit gewählte Angehörige des Bundespersonals, wie etwa Staatsanwälte des Bundes, sind Angestellte. ${ }^{10}$

Die rechtliche Stellung des Bundespersonals ist vornehmlich durch das BPG ${ }^{11}$ geprägt; bei einigen dezentralisierten Verwaltungseinheiten erweitert durch Sondernormen, wie etwa durch das IGEG ${ }^{12}$ für das Personal des Eidgenössischen Instituts für Geistiges Eigentum oder durch eine Verordnung ${ }^{13}$ für das Personal des Schweizerischen Heilmittelinstituts. Das Arbeitsverhältnis ist öffentlich-rechtlicher Natur. ${ }^{14}$ Das zivilrechtliche Obligationenrecht ist nur entsprechend anwendbar. ${ }^{15}$ Nur in Einzelfällen können Arbeitsverhältnisse zur Gänze zivilrechtlich begründet werden. ${ }^{16}$ Diese Ausnahme gilt aber nicht überall; so wird sie für das Personal des Eidgenössischen Instituts für Geistiges Eigentum wiederum grundsätzlich nicht angewendet. ${ }^{17}$ Das Arbeitsverhältnis kann unbefristet oder befristet abgeschlossen werden; ${ }^{18}$ in der Regel wird es unbefristet abgeschlossen. ${ }^{19}$ Auch die Wahl auf Zeit ist, wenngleich nur bei bestimmten Personalkategorien, möglich. ${ }^{20}$ Das Arbeitsverhältnis endet durch Erreichen der Altersgrenze, durch Invalidität, nach Ablauf der Befristung oder der Amtsdauer, durch gegenseitiges Einvernehmen oder durch Kündigung. ${ }^{21}$

6 Für die Schweiz gibt es kein für den gesamten öffentlichen Dienst geltendes Dienstrecht. Das Dienstrecht ist förderalistisch gegliedert; die Kantone und teilweise auch die Gemeinden kennen eigene Regelungen.

7 Auch die Kantone kennen bis auf wenige Ausnahmen eine solche Unterscheidung nicht mehr. Für die ersten Kantone, die den Beamtenstatus abgeschafft haben, vgl. Dommann, Personalrecht für Mitarbeitende im öffentlichen Dienst des Kantons Luzern; Lang, Das Zürcher Personalgesetz vom 27. September 1998; von Kaenel, Die Entwicklung des bernischen Dienstrechts; alle in: Helbling/Poledna (Hrsg.), Personalrecht (Fußn. 5), S. 35, 49, 79.

8 Hänni, Personalrecht des Bundes, 2. Aufl. 2002, Rdnr. 33.

9 Die altrechtlichen Dienstverhältnisse nach dem Beamtengesetz wurden in Angestelltenverhältnisse per 1. Januar 2002 überführt; für einige wenige Dienstverhältnisse gab es Übergangszeiten, vgl. Hänni, Personalrecht (Fußn. 8), Rdnr. 33.

10 Art. 1 Abs. 1 Verordnung über die auf Amtsdauer gewählten Angestellten vom 17. Oktober 2001 (SR 172.220.111.6).

11 Bundespersonalgesetz vom 24. März 2000 (SR 172.220.1).

12 Bundesgesetz über Statut und Aufgaben des Eidgenössischen Instituts für Geistiges Eigentum vom 24. März 1995 (SR 172.010.31).

13 Verordnung für das Personal des Schweizerischen Heilmittelinstituts vom 28. September 2001 (SR 812.215.4).

14 Art. 8 Abs. 1 S. 1 BPG.

15 Art. 6 Abs. 2 BPG.

16 Art. 6 Abs. 5 und 6 BPG; Art. 6a BPG.

17 Art. 8 Abs. 1 IGEG.

18 Art. 9 Abs. 1-2 BPG.

19 Häfelin/Müller, Allgemeines Verwaltungsrecht, 4. Aufl. 2002, Rdnr. 1552.

20 Art. 9 Abs. 4-5 BPG.

21 Art. 10-12 BPG. 
Das Bundespersonal unterliegt bestimmten Pflichten, wie sie auch der Beamtenstatus kannte: Die Pflicht zur Dienstleistung beinhaltet auch, daß die Bekleidung öffentlicher Ämter nur teilweise mit der Tätigkeit des Bundespersonals vereinbar ist und grundsätzlich zu bewilligen ist. ${ }^{22}$ Von zentraler Bedeutung ist die Treuepflicht. Dazu gehört nicht nur, die berechtigten Interessen des Bundes zu wahren, sondern sich auch in- und außerhalb des Dienstes der Achtung und des Vertrauens würdig zu erweisen, welches die Stellung des Bundespersonals erfordert. ${ }^{23}$ Diese Pflichten finden ihre Grenze im Schutz der Grundrechte, wie sie jedem Bürger zustehen. ${ }^{24}$ Darüber hinaus kennt das BPG kein Disziplinarverfahren mehr; Fragen des Fehlverhaltens sind vielmehr im Rahmen einer Kündigung maßgebend. ${ }^{25}$

Die Rechte des Bundespersonals liegen vor allem in der Entlohnung. Dabei gilt kein Alimentationsprinzip, sondern vielmehr von Gesetzes wegen ${ }^{26}$ das Leistungsprinzip. ${ }^{27}$ Darüber hinaus hat das Bundespersonal Ansprüche auf Lohnfortzahlung im Krankheitsfalle oder bei Unfall, welche sich von Beschäftigten in der Privatwirtschaft deutlich unterscheiden. ${ }^{28}$

\section{Alterssicherung des Bundespersonals im Kontext allgemeiner sozialer Sicherung}

Die Alterssicherung des Bundespersonals untersteht keinem Sondersystem der sozialen Sicherheit. ${ }^{29}$ Dies galt schon vor dem Wegfall des Beamtenstatus. ${ }^{30}$ Die Alterssicherung des Bundespersonals unterliegt dem allgemeinen Alterssicherungssystem für Arbeitnehmer. Sie beruht folglich auf den drei Säulen der Alterssicherung.

\footnotetext{
22 Art. 23 BPG.

23 BGE $99 \mathrm{Ib} 129 \mathrm{ff}$.

24 Jaag/Müller/Tschannen, Bundesverwaltungsrecht (Fußn. 2), S. 6.

25 Häfelin/Müller, Allgemeines Verwaltungsrecht (Fußn. 19), Rdnr. 1563.

26 Art. 15 Abs. 1 BPG.

27 Hänni, Personalrecht (Fußn. 8), Rdnr. 121 ff.; Henneberger/Sudjana, Einflüsse des New Public Management auf das Dienst- und Haushaltsrecht: Ein Rechtsvergleich zwischen der Schweiz und Deutschland, ZBR 2006, S. 14, 16 ff.

28 Hänni, Personalrecht (Fußn. 8), Rdnr. $151 \mathrm{f}$.

29 Anderes gilt wiederum für die Magistratspersonen: Bundesgesetz über Besoldung und berufliche Vorsorge der Magistratspersonen vom 6. Oktober 1989 (SR 172.121) und Verordnung über Besoldung und berufliche Vorsorge der Magistratspersonen vom 6. Oktober 1989 (SR 172.121.1).

$30 \mathrm{Vgl}$. für die berufliche Vorsorge (2. Säule): Pfitzmann, Die öffentlich-rechtlichen Pensionskassen im BVG-Obligatorium, SZS 1985, S. 233.
} 


\section{Ausgestaltung der Alterssicherung des Bundespersonals}

\section{Das Drei-Säulen-Konzept der Alterssicherung}

Die drei Säulen der Alterssicherung ${ }^{31}$ sind die universalistisch angelegte Alters-, Hinterlassenen- und Invalidenversicherung (1. Säule, AHV), die betriebliche Vorsorge (2. Säule) und die Selbstvorsorge (3. Säule). Eingeführt wurde das Drei-SäulenKonzept $^{32}$ im Dezember 1972 durch den neu eingefügten Art. 34quater der alten Bundesverfassung ${ }^{33}$. Nach dessen Absatz 1 sorgt der Bund „für eine ausreichende Alters-, Hinterlassenen- und Invalidenvorsorge. Diese beruht auf einer eidgenössischen Versicherung, der beruflichen und Selbstvorsorge." Auch die neue Bundesverfassung umschreibt das Drei-Säulen-Prinzip in Art. 111 Absatz 1 BV34 im gleichen Sinne: „Der Bund trifft Maßnahmen für eine ausreichende Alters-, Hinterlassenen- und Invalidenvorsorge. Diese beruht auf drei Säulen, nämlich der eidgenössischen Alters-, Hinterlassenen- und Invalidenversicherung, der beruflichen Vorsorge und der Selbstvorsorge.“

Von herausragender Bedeutung ist das Zusammenspiel zwischen 1. und 2. Säule. Die Bundesverfassung gibt dies vor: Nach Artikel 113 Absatz 2 lit.b BV soll die berufliche Vorsorge ,zusammen mit der Alters-, Hinterlassenen- und Invalidenversicherung die Fortsetzung der gewohnten Lebenshaltung in angemessener Weise“ ermöglichen, während die genannte klassische Altersvorsorge der Alters-, Hinterlassenen- und Invalidenversicherung nach Art. 112 Absatz 2 lit.b. BV lediglich „den Existenzbedarf angemessen zu decken" hat. Im Gegensatz zu zahlreichen anderen nationalen betrieblichen Altersvorsorgesystemen hat die 2. Säule damit nicht die bloße Funktion einer zusätzlichen Absicherung, sondern ist als tragendes Element in der Alterssicherung konzipiert. Die 3. Säule als Selbstvorsorge hingegen ist darauf ausgerichtet, einen Vermögensaufbau für das Alter über das Gewohnte hinaus zu bilden. Sie wird steuerlich teilweise begünstigt, indem die aufgewendeten Beträge für die gebundene Kapitalanlage bis zu einer Maximalhöhe privilegiert werden. ${ }^{35}$ Da die 3. Säule auf Freiwilligkeit beruht und unabhängig vom Status eines Arbeitsverhältnisses und vom Mitwirken der Arbeitgeber gebildet wird, bleibt sie im Folgenden außer Betracht.

31 Zum Drei-Säulen-Prinzip: Ross, Die berufliche Vorsorge im Alterssicherungssystem der Schweiz: Rechtliche Grundlagen und Reformansätze, in: Hennion-Moreau/Kaufmann (Hrsg.), Les retraites d'entreprise en Europe, droits europeen et comparé, 2007, S. $284 \mathrm{ff}$.

32 Zur historischen Entwicklung der drei Säulen: Ross, Geschlechterdifferenzierung, Rentenalter und Hinterlassenenversorgung in der Schweiz (1855-2005), ZIAS 2007, S. $182 \mathrm{ff}$.

33 Vgl. den in die alte Bundesverfassung der Schweizerischen Eidgenossenschaft vom 29. Mai 1879 eingefügten Art. 34quater im vom Bundesrat vorbereiteten Bundesbeschluß (BBl 1973 I 77-79).

34 Bundesverfassung der Schweizerischen Eidgenossenschaft vom 18. April 1999 (SR 101).

35 Amschwand-Pilloud/Jungo/Maute, Assurance-vie et impôts: Guide pratique, 2005, S. 46-53. 


\section{Die AHV als Erste Säule und Basissicherung}

Die universalistisch angelegte Alters- und Hinterlassenenversicherung soll den Existenzbedarf decken und ist damit der Grundstein der Alterssicherung wie insbesondere der beruflichen Vorsorge. Die Alters- und Hinterlassenenversicherung für jedermann ist hauptsächlich im $\mathrm{AHVG}^{36}$ und der zugehörigen Verordnung AHVV ${ }^{37}$ geregelt. Versichert sind grundsätzlich alle Personen, die in der Schweiz als Arbeitnehmer oder Selbständige erwerbstätig sind im Alter zwischen 18 und 65 Jahren (64 Jahren bei Frauen), sowie alle Personen, die in der Schweiz zwar nicht arbeiten, aber dort ihren Wohnsitz haben, im Alter zwischen 21 und 65 Jahren (64 Jahren bei Frauen). Damit sind fast die gesamte erwachsene Wohnbevölkerung in der Schweiz und alle Erwerbstätigen, die zur Arbeit in die Schweiz pendeln, in der Alters- und Hinterlassenenversicherung für jedermann bis einschließlich des 65 . Lebensjahres obligatorisch versichert. 38

\section{a) Leistungen der AHV: Minimal- und Maximalrente}

Renten aus diesem System werden gezahlt bei Erreichen des Rentenalters (Männer: Vollendung des 65. Lebensjahrs; Frauen: Vollendung des 64. Lebensjahrs) ${ }^{39}$ sowie unter bestimmten Voraussetzungen beim Tod des (Ehe-)Partners. Die Rentenhöhe bei Erreichen des Rentenalters hängt grundsätzlich von der Beitragszeit und dem maßgeblichen durchschnittlichen Jahreseinkommen ab. Die Berechnung ${ }^{40}$ der Rentenhöhe weist aber eine Spezialität auf: Je nach Beitragsjahren gestaffelt, gibt es eine garantierte Minimalrente und eine gedeckelte Maximalrente gemäß Art. 34 und 38 AHVG. Wer die Beitragszeit von 44 Beitragsjahren erreicht, erhält als Minimalrente eine monatliche Pension in Höhe von $1140 \mathrm{CHF}$, wenn sein Jahresdurchschnittseinkommen unter 13.860 CHF lag, und erhält eine Maximalrente in Höhe von monatlich 2280 CHF, wenn sein Jahresdurchschnittseinkommen über 82.080 CHF lag. Dazwischen liegende Jahresdurchschnittseinkommen ergeben jeweils nach deren Höhe eine Monatsrente, die zwischen 1140 und 2280 CHF liegt. So erhält etwa ein Rentenberechtiger, dessen jährliches Durchschnittseinkommen bei 40.000 CHF lag, eine monatliche Pension in Höhe von 1733 CHF. Werden die 44 Beitragsjahre nicht erreicht, so sinken die Monatsrenten, also auch die Minimal- und Maximalrente, prozentual hinsichtlich der fehlenden Beitragsjahre gemäß den in Art. 52 AHVV vorgegeben Rentenskalen.

36 Bundesgesetz vom 20. Dezember 1946 über die Alters- und Hinterlassenenversicherung (SR 831.10).

37 Verordnung vom 31. Oktober 1947 über die Alters- und Hinterlassenenversicherung (SR 831.101).

$38 \mathrm{Zu}$ den wenigen Ausnahmen: Kieser, Alters- und Hinterlassenenversicherung: Kommentar, 2. Aufl. 2005, Art. 1a Rdnr. 4-24.

39 Art. 21 AHVG.

40 Zur Berechnung im Einzelnen siehe Widmer, Die Sozialversicherung in der Schweiz, 5. Aufl. 2005, S. 54-62, sowie Bundesamt für Sozialversicherungen, Rententabellen 2009, AHV/IV, 2008. 


\section{b) Finanzierung der $A H V$}

Die Alters- und Hinterlassenenversicherung ist durch die Umlageverfahrensdeckung geprägt. Die Versicherten haben gemäß Art. 3 AHVG grundsätzlich Beiträge zu entrichten. Erwerbstätige zahlen nach Art. 4 AHVG Beiträge auf ihr Einkommen. Für unselbständig Beschäftigte, also für das Bundespersonal, wird der „maßgebende Lohn“41 als Berechnungsgrundlage herangezogen. Auf diesen hat der unselbständig Beschäftigte und sein Arbeitgeber jeweils 4,2 Prozent Beitrag, also insgesamt 8,4 Prozent zu entrichten (Art. 5, 13 AHVG). Neben den Beiträgen wird die Alters- und Hinterlassenenversicherung gemäß Art. 103 AHVG durch die öffentliche Hand finanziert. 2007 wurden 20,5 Prozent der jährlichen Ausgaben der Versicherung durch Bund und Kantone finanziert und der Bund leistete darüber hinaus einen Sonderbeitrag von 179 Mio. CHF; 2008 finanzierte nur noch der Bund die AHV mit, und zwar in Höhe von 19,55 Prozent der Jahresausgaben. ${ }^{42}$

\section{Berufliche Vorsorge als Zweite Säule}

Die berufliche Vorsorge findet ihre hauptsächlichen Regelungen im $\mathrm{BVG}^{43}$, in den zugehörigen drei Verordnungen BVV-144, BVV-245 und BVV-346 sowie in der Sicherheitsfondsverordnung SFV 47 . Im Gegensatz zu den anderen klassischen Sozialversicherungsgesetzen, wie beim AHVG, findet der Allgemeine Teil des Sozialversicherungsgesetzes (ATSG) ${ }^{48}$ grundsätzlich keine Anwendung auf die berufliche Vorsorge. 49 Kernelement des BVG ist die Einführung eines Obligatoriums. Mit diesem werden bestimmte Lebenssachverhalte der beruflichen Vorsorge unterstellt. Das BVG enthält Mindestanforderungen an die berufliche Vorsorge, was nichts anderes heißt, als daß

41 Zur Bestimmung des maßgeblichen Lohns: Kieser, Alters- und Hinterlassenenversicherung (Fußn. 38), Art. 5 Rdnr. 92-150.

42 Kieser, Alters- und Hinterlassenenversicherung, in: Meyer (Hrsg.), Soziale Sicherheit, 2. Aufl. 2007, S. 1165, 1288; Bundesamt für Sozialversicherung, AHV-Statistik 2009, 2009, S. 4.

43 Bundesgesetz vom 25. Juni 1982 über die berufliche Alters-, Hinterlassenen- und Invalidenvorsorge (SR 831.40).

44 Verordnung vom 29. Juni 1983 über die Beaufsichtigung und die Registrierung der Vorsorgeeinrichtungen (SR 831.435.1).

45 Verordnung vom 18. April 1984 über die berufliche Alters-, Hinterlassenen- und Invalidenvorsorge (SR 831.441.1).

46 Verordnung vom 13. November 1985 über die steuerliche Abzugsberechtigung für Beiträge an anerkannte Vorsorgeformen (SR 831.461.3).

47 Verordnung vom 22. Juni 1998 über den Sicherheitsfonds BVG (SR 831.432.1).

48 Bundesgesetz vom 6. Oktober 2000 über den Allgemeinen Teil des Sozialversicherungsrechts (SR 830.1).

49 Kieser, ATSG-Kommentar: Kommentar zum Bundesgesetz über den Allgemeinen Teil des Sozialversicherungsrechts vom 6. Oktober 2000, 2003, Art. 2 Rdnr. 18. 
hiervon zu Gunsten der Versicherten abgewichen werden kann. 50 Über das Obligatorium hinaus ist das Konzept der beruflichen Vorsorge auch offen für andere, nicht dem Obligatorium unterliegende Lebenssachverhalte.

In der beruflichen Vorsorge werden obligatorisch nur Arbeitnehmerinnen und Arbeitnehmer versichert. Damit unterscheidet sich das Konzept der beruflichen Vorsorge deutlich von der 1. Säule der Alterssicherung, die für die fast gesamte Wohn- und Arbeitsbevölkerung gilt. Aber nicht jede unselbständige Beschäftigung unterliegt der obligatorischen beruflichen Vorsorge. Zum verbindlichen Versichertenkreis ${ }^{51}$ gehören nach Art. 2 und 7 BVG nur diejenigen, deren pflichtiger Jahreslohn nach dem AHVG höher ist als Dreiviertel der Maximalrente nach dem AHVG, demnach bei einem Jahreslohn von über 20.520 CHF, die diesen Jahreslohn bei einem Arbeitgeber verdienen, die einen unbefristeten oder einen über drei Monate hinaus befristeten Arbeitsvertrag haben und die das 17. Lebensjahr vollendet und das Rentenalter noch nicht erreicht haben, wobei die 18- bis 24jährigen nur gegen die Risiken Tod oder Invalidität und alle anderen auch gegen das Risiko Alter versichert werden. Ausgeschlossen werden von diesem Versichertenkreis diejenigen, deren Arbeitgeber nach dem AHVG nicht beitragspflichtig sind, die nebenberuflich tätig und bereits für eine hauptberufliche Tätigkeit obligatorisch versichert sind, die im Sinne der Invalidenversicherung zu mindestens 70 Prozent invalide sind, oder die als Familienmitglieder in landwirtschaftlichen Betrieben arbeiten. ${ }^{52}$ Insgesamt werden in der beruflichen Vorsorge somit obligatorisch nur solche Arbeitnehmerinnen und Arbeitnehmer unterstellt, deren jetziges Einkommen zumindest in der Nähe der Maximalrente nach dem AHVG liegt. Damit soll verhindert werden, daß diejenigen, die schon heute ein Einkommen haben, welches unter demjenigen liegt, was man für sie als angemessenen Existenzbedarf im Alter vorgesehen hat, mit weiteren Abgaben belastet werden.

\section{a) Einrichtungen der Beruflichen Vorsorge des Bundespersonals}

Jeder Arbeitgeber, der obligatorisch Versicherte beschäftigt, muß eine Einrichtung der beruflichen Vorsorge errichten oder sich einer bestehenden anschließen ${ }^{53}$, auch Sammeleinrichtungen mehrerer Arbeitgeber sind möglich. Für das Bundespersonal gibt es einerseits die Sammeleinrichtung Publica, die verschiedene Vorsorgewerke beherbergt, und andererseits eigenständige Einrichtungen wie etwa die Pensionskasse der Schweizerischen Bundesbahn (SBB) oder diejenige der Post.

50 Widmer, Sozialversicherung (Fußn. 40), S. 128.

51 Zum obligatorischen Versichertenkreis im Einzelnen: Stauffer, Die Berufliche Vorsorge: BVG/FZG/ZGB/OR/FusG, 2. Aufl. 2006, S. 4-8, 11-13 und 16-20; Vetter-Schreiber, Berufliche Vorsorge: Kommentar, 2005, S. 39-43, 55-57 und 60-63.

52 Art. 1j BVV-2.

53 Art. 11 Abs. 1 BVG. 
Eine Einrichtung der beruflichen Vorsorge muß eine rechtlich selbständige Institution sein. Als Rechtsform ist zwingend die Stiftung, die Genossenschaft oder eine Einrichtung des öffentlichen Rechts vorgeschrieben. ${ }^{54}$ Die Einrichtungen der beruflichen Vorsorge haben Statuten zu erlassen, ${ }^{55}$ welche die Leistungen, die Organisation, die Verwaltung, die Finanzierung und die Kontrolle regeln. Jede Einrichtung legt somit fest, ob sie über die Mindestvorschriften des BVG hinausgeht, zum Beispiel indem sie sich für das Leistungsprimat entscheidet.

\section{b) Leistungen der Beruflichen Vorsorge: Kapitalgedeckte Altersrente}

Das System der beruflichen Vorsorge erbringt Leistungen für Altersrentenberechtigte, aber auch für die sogenannten Hinterlassenen, Verwitwete und Waisen, und für die Invaliden. Die Leistungen sind immer Geldleistungen. Die Geldleistungen im Alter werden in der Regel als Rente ausgezahlt und können unter bestimmten Bedingungen auch als Kapitalabfindung ausgerichtet werden.

Wie die Altersrente berechnet wird, hängt zunächst davon ab, welchen Typ der Rente die jeweilige Einrichtung der beruflichen Vorsorge vorgegeben hat. Man unterscheidet dabei das Beitragsprimat vom Leistungsprimat. Das Beitragsprimat orientiert sich grundsätzlich an der Addition der Altersgutschriften und ihrer Verzinsung, woraus die Renten gebildet werden. Das Leistungsprimat orientiert sich an einer bestimmten prozentualen Höhe der Rente, beispielsweise 60 Prozent des letzten Lohns. Der Unterschied liegt darin, daß sich die Rente nach dem Beitragsprimat am koordinierten Lohn ausrichtet und somit an den Schwankungen im Einkommen. Das Leistungsprimat sieht hingegen eine bestimmte Rente vor, gibt also garantierte Höhen vor. Das Beitragsprimat ist eine Mindestanforderung des BVG. Das Leistungsprimat ist hingegen eine Option, die zu Gunsten der Versicherten über die Mindestanforderung hinaus geht. Im Jahre 2007 wurden 2,9 Mio. Versicherte nach dem Beitragsprimat und 0,6 Mio. nach dem Leistungsprimat versichert. 56

Für Beschäftigte im öffentlichen Dienst ergab sich aber ein ganz anderes Verhältnis: Die öffentlich-rechtlichen Einrichtungen versicherten 0,21 Mio. nach dem Beitragsprimat und 0,35 Mio. nach dem Leistungsprimat. ${ }^{57}$ Damit kann zu diesem Zeitpunkt von einem tendenziellen Übergewicht der Rentenberechnung anhand des letzten Lohns für Beschäftigte im öffentlichen Dienst gesprochen werden. Für Angehörige des Bundespersonals kann dies ab dem Jahre 2008 nicht mehr gelten: Alle Vorsorgereglements der

54 Art. 48 Abs. 2 BVG.

55 Art. 50 BVG.

56 Bundesamt für Statistik (Hrsg.), Die berufliche Vorsorge in der Schweiz: Pensionskassenstatistik 2007, 2009, S. 13.

57 Ibidem. 
Vorsorgewerke von Publica haben vom Leistungsprimat auf das Beitragsprimat umgestellt ${ }^{58}$, gleiches gilt für die Pensionskassen der $\mathrm{SBB}^{59}$ und der Post ${ }^{60}$.

Wie die Renten nach dem Beitragsprimat mindestens, das heißt nach verbindlicher Mindestformel, zu berechnen sind, geben die Art. 14-16 BVG vor: Zunächst werden die Altersgutschriften gebildet. Diese orientieren sich jeweils am versicherten koordinierten Lohn eines Beitragsjahres. Von diesem wird ein Betrag für die Altersgutschrift entnommen. Dies geschieht dadurch, daß man von den koordinierten Löhnen bestimmte Beträge für bestimmte Altersklassen ansetzt. Bei den 25 bis 34jährigen werden sieben Prozent des jeweiligen koordinierten Lohnes eines Beitragsjahres entnommen, bei den 35 bis 44jährigen zehn Prozent, bei den 45 bis 54jährigen 15 Prozent und bei den 55 bis 65jährigen 18 Prozent. Hat ein 50jähriger einen versicherten koordinierten Lohn von 30.000 CHF im Jahr 2010, so werden ihm für das Jahr 201015 Prozent des koordinierten Lohns gutgeschrieben, also 4500 CHF.

Für das Bundespersonal wird von der genannten Mindestformel zu Gunsten der Versicherten erheblich abgewichen, was nicht zuletzt mit den höheren Beiträgen, insbesondere den teilweise höheren Arbeitgeberbeiträgen, zusammenhängt. So werden beim Vorsorgewerk Bund, bei dem etwa die Angestellten der Departements und Ämter versichert sind, hinsichtlich der unteren Lohngruppen bei den 25 bis 34jährigen elf Prozent des jeweiligen koordinierten Lohnes eines Beitragsjahres entnommen, bei den 35 bis 44jährigen 14 Prozent, bei den 45 bis 54jährigen 20,5 Prozent und bei den 55 bis 65jährigen 27 Prozent. ${ }^{61}$ Beim Vorsorgewerk Eidgenössisches Institut für Geistiges Eigentum werden bei den 25 bis 34jährigen elf Prozent des jeweiligen koordinierten

58 Vgl. Art. 36 ff. Vorsorgereglement für die Angestellten und Rentenbeziehenden des Vorsorgewerkes Bund vom 15. Juni 2007; Art. 36 ff. Vorsorgereglement des Vorsorgewerks ETH-Bereich für die Mitarbeiterinnen und Mitarbeiter des ETH-Bereich vom 9. November 2007; Art. 36 ff. Vorsorgereglement des Vorsorgewerks ETH-Bereich für die Professorinnen und Professoren des ETHBereich vom 9. November 2007; Art. 36 ff. Vorsorgereglement für die Angestellten und die Rentenbeziehenden des Vorsorgewerks Angeschlossene Organisationen vom 14. März 2008; Art. 36 ff. vom 21. November 2007; Art. 35 ff. Vorsorgereglement für die Angestellten und die Rentenbeziehenden des Vorsorgewerks IGE (Eidgenössisches Institut für Geistiges Eigentum) vom 21. November 2007; Art. 36 ff. Vorsorgereglement für die Angestellten und die Rentenbeziehenden des Vorsorgewerks der Eidgenössischen Revisionsaufsichtsbehörde RAB vom 20. November 2007; Art. 36 ff. Vorsorgereglement für die Angestellten und die Rentenbeziehenden des Vorsorgewerks des Eidgenössischen Hochschulinstituts für Berufsbildung EHB vom 15. November 2007; Art. 36 ff. Vorsorgereglement für die Angestellten und Rentenbeziehenden des Vorsorgewerks Swissmedic vom 21. September 2007; Art. 37 ff. Vorsorgereglement für die Angestellten und Rentenbeziehenden des Vorsorgewerks FINMA vom 12. Dezember 2008; alle im Internet unter: http://www.publica.ch/page/content/index.asp?MenuID=186\&ID=256\&Menu=1\&Item=24.

59 Art. 28 Vorsorgereglement der Pensionskasse SBB vom 1. Januar 2007; abrufbar im Internet unter: http://www.pksbb.ch/cms/media.php?id=70\&cat=28.

60 Art. 37 Vorsorgereglement der Pensionskasse Post vom 1. Januar 2008; abrufbar im Internet unter: http://www.pkpost.ch/de/pdf/Vorsorgereglement_d.pdf.

61 Vgl. Art. 24 Vorsorgereglement Vorsorgewerk Bund (Fußn. 58). 
Lohnes eines Beitragsjahres entnommen, bei den 35 bis 44jährigen 15 Prozent, bei den 45 bis 54jährigen 23,5 Prozent und bei den 55 bis 65jährigen 31,5 Prozent. ${ }^{62}$

Aus der Summe der jährlichen Gutschriften, zuzüglich der Verzinsung, wird die Altersgutschrift gebildet. Weiterhin können noch, je nach Statut der Einrichtung der beruflichen Vorsorge, partielle Anteile am überschießenden Gewinn aus der Kapitalanlage in die individuelle Altersgutschrift einfließen. Zur Ermittlung der monatlichen Rente wird die Altersgutschrift mit dem Rentenumwandlungssatz multipliziert, der zurzeit noch bei 0,068 liegt, ${ }^{63}$ und durch zwölf geteilt. Beläuft sich für eine Versicherte beim Erreichen des Rentenalters die Altersgutschrift auf $250.000 \mathrm{CHF}$, so ergibt sich für sie eine monatliche Rente aus der beruflichen Vorsorge in Höhe von 1417 CHF $(=(250.000$ CHF x $0,068) . / .12)$.

\section{c) Leistungen der Beruflichen Vorsorge: Kapitalabfindung}

Die Versicherten können sich einen Teil des Altersguthabens als Kapitalabfindung auszahlen lassen. Bei der Berechnung der Altersrente vermindert sich dann die Altersgutschrift um diesen Betrag. Nach Art. 37 Abs. 2 BVG kann jeder Versicherte ein Viertel des Altersguthabens verlangen. Da dies nur eine Mindestvorschrift ist, kann hiervon abgewichen werden. Angehörige des Bundespersonals, die in einem Vorsorgewerk der Sammeleinrichtung Publica versichert sind, können aufgrund der diesbezüglich gleich lautenden Reglements immer bei Erreichen des Rentenalters die Hälfte des Altersguthabens als Kapitalabfindung beanspruchen; wünschen sie 51 bis 100 Prozent, müssen sie drei Jahre vor Erreichen des Rentenalters einen Antrag stellen. ${ }^{64}$ Bei der Pensionskasse SBB können 50 Prozent bezogen werden, nur Angestellte in hohen Lohnklassen können bis zu 100 Prozent verlangen. ${ }^{65}$ Versicherte der Pensionskasse Post können sich immer bis zu 100 Prozent der Altersgutschrift als Kapitalabfindung auszahlen lassen, wenn sie dies bis zu drei Monate vor Erreichen der Altergrenze beantragen. 66

Auch während des Erwerbslebens darf Kapital aus der Vorsorgeeinrichtung entnommen werden, wenn bis drei Jahre vor der Pensionierung selbstgenutztes Wohneigentum finanziert werden soll. Nach der WEFV 67 werden solche Entnahmemöglichkeiten geregelt. Grundsätzlich muß der entnommene Betrag zur Finanzierung von Wohneigentum verwendet werden. Damit kein Mißbrauch entstehen kann, wird nach Art. 30d BVG im Grundbuch eine Veräußerungsbeschränkung eingetragen. Dadurch wird ver-

62 Vgl. Art. 23 Vorsorgereglement Vorsorgewerk IGE (Fußn. 58).

63 Art. 14 Abs. 2 BVG.

64 Statt aller vgl. Art. 40 Vorsorgereglement Vorsorgewerk Bund (Fußn. 58) und Art. 39 Vorsorgereglement Vorsorgewerk IGE (Fußn. 58).

65 Art. 30 Abs. 1 Vorsorgereglement Pensionskasse SBB (Fußn. 58).

66 Art. 50 Vorsorgereglement Pensionskasse Post (Fußn. 58).

67 Verordnung vom 3. Oktober 1994 über die Wohneigentumsförderung mit Mitteln der beruflichen Vorsorge (SR 831.411). 
hindert, daß der Versicherte sein Wohneigentum veräußert und den Erlös nicht wieder an die Vorsorgeeinrichtung abführt.

\section{d) Finanzierung der Beruflichen Vorsorge für das Bundespersonal}

Die berufliche Vorsorge wird hauptsächlich durch Beiträge finanziert. Zuschüsse aus öffentlichen Mitteln, sieht man einmal von steuerlichen Privilegierungen ab, sind dem System der beruflichen Vorsorge im Grunde fremd. Die Höhe der Beiträge ist im Gegensatz zu anderen Sozialversicherungssystemen, insbesondere der Alters- und Hinterlassenenversicherung, nicht vorgegeben. Sie wird von jeder Einrichtung der beruflichen Vorsorge festgelegt. Da an diese aber Mindestanforderungen gestellt werden, wie etwa die Sicherung für die Leistungen im Alter und die grundsätzliche Kapitaldeckung der beruflichen Vorsorge, ergibt sich schon faktisch die Konsequenz, daß die Beiträge für die gesamte Versicherungszeit durchschnittlich ungefähr bei mindestens 17 bis 18 Prozent des koordinierten Lohns liegen müssen. ${ }^{68}$ Dies gilt insbesondere bei denjenigen Einrichtungen der beruflichen Vorsorge, die ihr System an der Mindestanforderung des Beitragsprimats ausgerichtet haben.

In der beruflichen Vorsorge wird nicht der gesamte Lohn obligatorisch versichert, sondern nur der sogenannte koordinierte Lohn nach Art. 8 BVG. Dieser ist die Differenz zwischen dem tatsächlichen Jahreslohn und sieben Achtel der Maximaljahresrente nach AHVG (Koordinationsabzug), also gegenwärtig 23.940 CHF. Die Differenz ist bis zu einer Höchstgrenze von 58.140 CHF obligatorisch zu versichern. Verdient ein Arbeitnehmer im Jahr 40.000 CHF, so sind hiervon 23.940 CHF abzuziehen, sodaß 16.060 CHF zu versichern sind. Verdient eine Arbeitnehmerin im Jahr 90.000 CHF, so sind hiervon ebenfalls 23.940 CHF abzuziehen; der sich ergebende Differenzwert von 66.060 CHF übersteigt aber die Höchstgrenze des Obligatoriums, sodaß in diesem Fall der zu versichernde koordinierte Lohn nur die Höchstgrenze, also 58.140 CHF beträgt. Der koordinierte Lohn ist die Grundlage zur Ermittlung der zu zahlenden Beiträge und der Altersgutschriften im Leistungsfall. Besonders aus letzterem Gesichtspunkt wurde eine Fiktion bei der Ermittlung des koordinierte Lohns eingeführt: Bei Jahresverdiensten, die zwar personell die Versicherungspflicht auslösen, aber unterhalb des Koordinationsabzuges liegen, demnach zwischen 20.520 und 23.940 CHF, wird ein koordinierender Lohn von $3420 \mathrm{CHF}$ als Berechnungsgrundlage angesetzt. Damit wurde auch für diese Einkommen die Voraussetzung geschaffen, von der beruflichen Vorsorge Gebrauch zu machen.

Die Beiträge sind vom Arbeitgeber an die Einrichtung der beruflichen Vorsorge abzuführen. Getragen werden sie mindestens zur Hälfte vom Arbeitgeber. ${ }^{69}$ Der Rest wird

68 Helbling, in: ders. (Hrsg.), Personalvorsorge und BVG: Gesamtdarstellung der rechtlichen, betriebswirtschaftlichen, organisatorischen und technischen Grundlagen der beruflichen Vorsorge in der Schweiz, 8. Aufl. 2006, S. 173-176.

69

Art. 66 Abs. 1 BVG. 
den versicherten Personen auferlegt. Das genaue Verhältnis zwischen den Arbeitgeberund den Arbeitnehmerbeiträgen legt wiederum die Einrichtung der beruflichen Vorsorge fest. Dabei wird häufig der Arbeitgeberanteil höher als der mindestens hälftige Anteil festgesetzt. Im Jahre 2007 standen Arbeitgeberbeiträge in Höhe von 19 Mrd. CHF Arbeitnehmerbeiträge in Höhe von 13,8 Mrd. CHF gegenüber. ${ }^{70}$ Beim Bundespersonal ist der Beitrag des Arbeitgebers grundsätzlich höher als derjenige des Arbeitnehmers. Beim Vorsorgewerk Bund sind die Sparbeiträge nur für Jüngere gleich, für Ältere übernimmt der Arbeitgeber einen größeren Anteil. Darüber hinaus wird die besondere Risikoprämie allein vom Arbeitgeber übernommen. ${ }^{71}$ Auch beim Vorsorgewerk Eidgenössisches Institut für Geistiges Eigentum übernimmt der Arbeitgeber nicht nur die Risikoprämie, sondern bezahlt für Angestellte ab dem 45. Lebensjahr 56 Prozent und bei Angestellten ab dem 55. Lebensjahr 57 Prozent der Beiträge. 72

Die berufliche Vorsorge wird aber nicht nur durch die Beiträge finanziert. Um die Mindestanforderungen an die Sicherung der Leistungen im Alter zu gewährleisten und um insbesondere der vorgegebenen Mindestverzinsung der Altersgutschriften zu entsprechen, legen die Einrichtungen der beruflichen Vorsorge Kapital an, um Erträge aus den Beiträgen zu erwirtschaften. Von den mannigfaltigen Anlagemöglichkeiten werden insbesondere Obligationen, Aktien und Immobilien bevorzugt. Im Jahre 2007 betrug die Summe der direkten und kollektiven Anlagen insgesamt ein Vermögen in Höhe von 602,6 Mrd. CHF; hiervon wurden 37 Prozent in Obligationen, 27,8 Prozent in Aktien und 14,4 Prozent in Immobilien angelegt. ${ }^{73}$

\section{e) Sicherung der Beruflichen Vorsorge für das Bundespersonal}

Die Einrichtungen der beruflichen Vorsorge, insbesondere ihr Anlageverhalten, unterliegen einem facettenreichen Kontrollwesen. Jede Einrichtung muß eine Kontrollstelle nach Art. 53 Absatz 1 BVG errichten, deren Aufgabe es ist, jährlich die Geschäftsführung, das Rechnungswesen und die Vermögensanlage zu prüfen. Darüber hinaus muß jede Einrichtung einen Experten der beruflichen Vorsorge berufen, der nach Art. 53 Absatz 2 BVG die finanzielle Sicherheit für die Leistungen im Alter und die Übereinstimmung der Statuten mit dem BVG zu überwachen hat. Die Einrichtungen der beruflichen Vorsorge werden auch extern beaufsichtigt. Einrichtungen, die in der gesamten Schweiz operieren, werden vom Bundesamt für Sozialversicherung nach Art. 3 BVV-1 und alle anderen von kantonalen Aufsichtsbehörden nach Art. 2 BVV-1 beaufsichtigt. Nach Art. 62 BVG sollen diese Behörden die Statuten, insbesondere über die Anlage des Vermögens, und die Geschäftstätigkeit überwachen.

70 Bundesamt für Statistik (Hrsg.), Pensionskassenstatistik 2007 (Fußn. 56), S. 23.

71 Art. 24 und 26 Vorsorgereglement Vorsorgewerk Bund (Fußn. 58).

72 Art. 23 und 25 Vorsorgereglement Vorsorgewerk IGE (Fußn. 58).

73 Bundesamt für Statistik (Hrsg.), Pensionskassenstatistik 2007 (Fußn. 56), S. 15 f. 
Weiterhin kennt das System der beruflichen Vorsorge nach Art. 56 BVG den sogenannten Sicherheitsfonds. Dieser wird von den Spitzenverbänden der Arbeitnehmer und der Arbeitgeber errichtet. Der Fonds hat zwei wichtige Aufgaben: Zum einen richtet er Zuschüsse an solche Einrichtungen aus, die eine ungünstige Altersstruktur haben, also viele ältere Arbeitnehmer und Arbeitnehmerinnen versichern. Getragen werden diese Zuschüsse durch Beiträge, welche die Einrichtungen der beruflichen Vorsorge an ihn erbringen. Zum anderen dient der Sicherheitsfonds der Sicherstellung der Leistungen im Alter für solche Einrichtungen, die insolvent geworden sind. Sichergestellt werden dabei nur die gesetzlichen Leistungen, das heißt die Mindestanforderungen des BVG. Die zu schaffenden Reserven für diese Funktion werden ebenfalls durch Beiträge aller Vorsorgeeinrichtungen aufgebracht. ${ }^{74}$

Der Bund übernimmt heute für die berufliche Vorsorge seines Bundespersonals nur noch teilweise eine Letztverantwortung - einerseits wegen des Vorhandenseins des Sicherheitsfonds, andererseits aufgrund bereits getätigter Zuschüsse, so etwa bei der Errichtung der Sammeleinrichtung Publica, wo er Fehlbeträge in Höhe von 11,9 Mrd. CHF der vormaligen Pensionskasse Bund übernommen hat oder für Härtefälle derjenigen Organisationen, die sich Publica angeschlossen haben, eingestanden ist, ${ }^{75}$ oder das Betriebskapital von Publica als Anschubfinanzierung übernommen hat ${ }^{76}$.

\section{Bewertung}

Das Bundespersonal besteht aus Angestellten. Beamte gibt es nicht mehr. Einige Pflichten erinnern noch an Pflichten aus dem Beamtenstatus; doch zeigt schon die Entlohnung nach dem Leistungsprinzip, daß das Bundespersonal sich vom Personal in der Privatwirtschaft kaum noch unterscheidet. Das Bundespersonal ist in die allgemeine Alterssicherung für Arbeitnehmer integriert. Ihre Absicherung erfolgt dabei nach den Grundsätzen der 1. und 2. Säule der allgemeinen Alterssicherung.

Die Regeln der Alters- und Hinterlassenversicherung (1. Säule) lassen für das Bundespersonal keinen Raum für Sondertatbestände. Die von Verfassung wegen vorgegebene Funktion der 1. Säule, den Existenzbedarf, und nur diesen, im Alter zu gewährleisten, führt dazu, daß die Altersleistungen der 1. Säule immer Renten sind. Kapitalabfindungen sind nicht möglich. Die Altersleistungen orientieren sich in ihrer Höhe an den im Erwerbsleben eingezahlten Beiträgen zur 1. Säule. Dem Verfassungsauftrag gemäß resultiert aus der 1. Säule eine Mindestrente nach Erreichen einer bestimmten Anzahl von Beitragsjahren. Zur solidarischen Finanzierung der Existenzbedarfsdeckungsrente

74 Hierzu insgesamt: Lüönd, Der Sicherheitsfonds BVG, 2004.

75 Art. 19 Abs. 1 und Abs. 3 Bundesgesetz über die Pensionskasse des Bundes (Publica-Gesetz) vom 20. Dezember 2006 (SR 172.222.1).

76 Art. 22 ibidem. 
gehört es auch, daß es eine Maximalrente gibt, unabhängig von der Höhe der tatsächlich gezahlten Beiträge. Die Beiträge werden immer vom Arbeitnehmer und vom Arbeitgeber zur Hälfte getragen. Aufgrund der besonderen Funktion der 1. Säule übernimmt die öffentliche Hand einen Teil ihrer Finanzierung.

Die Berufliche Vorsorge (2. Säule) läßt grundsätzlich Raum für Sonderregeln für das Bundespersonal. Davon wird aber nur teilweise Gebrauch gemacht. Die 2. Säule soll zusammen mit der 1. Säule von Verfassung wegen es ermöglichen, die gewohnte Lebenshaltung in angemessener Weise fortzuführen. Der 2. Säule kommt für das Bundespersonal also eine herausragende Stellung zu. Altersleistungen können Renten und Kapitalabfindung sein. Beide Leistungen orientieren sich an der Altersgutschrift, welche sich an bestimmten Summen aus vorausgegangenen Löhnen und Zinserträgen daraus zusammensetzt. Von der Möglichkeit, die Altersleistungen am letzten oder an den letzten Löhnen zu orientieren, wird für das Bundespersonal kein Gebrauch mehr gemacht. Die Summen, die zur Altersgutschrift für das Bundespersonal führen, übertreffen jene, welche das Gesetz mindestens vorgibt. Dies rührt vor allem aus den hohen Beiträgen. Diese müßten zwar nur zur Hälfte vom Arbeitgeber getragen werden, doch zeigt es sich, daß beim Bundespersonal der Arbeitgeber immer mehr als die Hälfte übernimmt. Die Kapitalabfindung, die sich ebenfalls an der Altersgutschrift orientiert, kann beim Großteil des Bundespersonals bis zu einer Höhe von 100 Prozent der Altersgutschrift ausgezahlt werden. Damit wird das Risiko der Verwendung dieses Kapitals in die Hände der Angestellten gelegt, was nicht zuletzt dem Verfassungsgebot der Eigenverantwortung entspricht, und nur deswegen gewährt wird, weil die 1. Säule dies nicht kennen kann, dient sie doch der Existenzbedarfsdeckung, welche aus Renten herrühren muß. Die Finanzierung der 2. Säule basiert darauf, daß die Beiträge angelegt werden. Zur Sicherstellung, daß mit der 2. Säule die gewohnte Lebenshaltung in angemessener Weise fortgeführt werden kann, sind zahlreiche Kontrollmechanismen eingeführt worden. Das Anlageverhalten der Einrichtungen der 2. Säule wird intern wie extern kontrolliert. Darüber hinaus werden finanzielle Einbußen von einem eigenständigen Fonds aufgefangen. Schließlich hat der Bund zur Sicherstellung der Finanzierung der 2. Säule für das Bundespersonal beträchtliches Kapital zur Verfügung gestellt. ${ }^{77}$

Die Alterssicherung des Bundespersonals wird insgesamt durch zwei Elemente geprägt: Starre Regeln und geringes Risiko dort, wo es um die Sicherung der Existenzbedarfsdeckung geht. Flexiblere Regeln und höheres Risiko dort, wo es um die Sicherung der Fortführung der gewohnten Lebenshaltung geht. Letztere wird durch zahlreiche Maßnahmen flankiert, welche die Risiken mindern. Ein wesentliches Prinzip der Alterssicherung des Bundespersonals ist die Risikominimierung hinsichtlich des Ertrages aus der Altersvorsorge.

77 Zur Sicherstellung der 2. Säule angesichts der Finanzmarktkrise vgl. Ross, Die obligatorische berufliche Vorsorge in der Schweiz: Betriebliche Alterssicherung mit Verfassungsauftrag, Soziale Sicherheit 12/2009, S. $423 \mathrm{ff}$. 


\title{
Die Alterssicherung von Beamten in Slowenien
}

\author{
Grega Strban
}

I. Einleitung

II. Der öffentliche Dienst im slowenischen Rechtssystem

III. Die Rechtsstellung der Beschäftigten im öffentlichen Dienst

1. Verfassungsrechtliche Vorgaben

2. Die monistische Konzeption des Arbeitsrechts

3. Allgemeine Grundsätze des Beamtensystems

4. Das Beamtenverhältnis
a) Abschluß eines Dienstvertrages
b) Inhalt des Dienstvertrags
c) Auflösung des Dienstvertrags
d) Rechtsweg

IV. Die Altersversorgung von Beamten

1. Das Verfassungsrecht

2. Die verschiedenen Altersvorsorgesysteme für Beamte

a) Die gesetzliche Rentenversicherung

aa) Allgemeiner Geltungsbereich

bb) Leistungen

cc) Finanzierung und Verwaltung

b) Die obligatorische Alterszusatzversicherung

c) Die freiwillige Alterszusatzversicherung

d) Die kollektive Alterszusatzversicherung für Beamte

e) Die staatliche Rente

V. Abschließende Bemerkungen und die weitere Entwicklung de lege ferenda 